\title{
基于三维手部骨架数据的连续手语识别
}

\author{
王卓程, 张景峤* \\ (上海大学计算机工程与科学学院 上海 200444) \\ (jqzhang@shu.edu.cn)
}

\begin{abstract}
摘 要: 为有效地消除手语识别过程中背景、光照等干扰因素带来的视觉问题, 采用低冗余的骨架数据表达手语信 息，设计了一个端到端连续手语识别模型. 首先，分别从帧内和帧间提取手型和轨迹特征，可以有效地降低原始样本 的离散程度; 其次, 构建一系列并行的双路残差网络对手型和轨迹特征进行优化与融合, 生成时空特征序列; 最后, 基于注意力机制的编码-解码网络实现时空特征序列到翻译文本的映射. 使用 Leap Motion 收集建立了一个基于三维 手部骨架数据的手语数据集 LMSLR. 实验结果表明, 在 LMSLR 数据集和公共的 CSL 数据集上, 该模型与大多数基 于视频处理的模型相比具有较高的准确率和较小的计算量.
\end{abstract}

关键词: 手语识别; 骨架数据; 残差网络; 注意力机制

中图法分类号: TP391.41 DOI: 10.3724/SP.J.1089.2021.18816

\section{Continuous Sign Language Recognition Based on 3D Hand Skeleton Data}

\author{
Wang Zhuocheng and Zhang Jingqiao* \\ (School of Computer Engineering and Science, Shanghai University, Shanghai 200444)
}

\begin{abstract}
In sign language recognition, it is necessary to eliminate the visual problems caused by interference factors such as background and light. Therefore, an end-to-end continuous sign language recognition model is designed using low-redundant skeleton data. Firstly, the shape and trajectory features are extracted from intra frame and inter frame respectively, which can reduce the discreteness of the original samples. Secondly, a series of parallel two-stream residual networks are constructed to fuse shape and trajectory features, further generate the spatial-temporal feature sequence. Finally, the attention-based encoder-decoder network is used to realize the mapping of the fused feature sequence to the translated text. In addition, a new skeleton-based sign language dataset using Leap Motion is collected named LMSLR. Experimental results on the LMSLR dataset and the public CSL dataset show that the proposed model has higher accuracy and lower computational complexity than most models based on video processing.
\end{abstract}

Key words: sign language recognition; skeleton data; residual network; attention mechanism

手语是为袭哑群体设计的一种视觉语言, 而 手语识别是利用计算机技术将手语翻译成文本语 言的技术手段. 手语识别技术的作用是多方面的. 首先，它将大大改善狵哑群体的交流状态，特别是
解决狵哑人与正常人之间的语言障碍; 其次, 手语 识别是涉及计算机图形学、计算机视觉、人机交互、 自然语言处理等多学科的交叉研究领域, 因此对 手语识别的研究有利于推动相关学科的发展.

收稿日期: 2020-11-30; 修回日期: 2021-03-02. 基金项目：上海市自然科学基金(19ZR1419200). 王卓程(1996一), 男, 硕士研究 生, CCF 学生会员, 主要研究方向为手语识别、深度学习; 张景峤(1975一), 女, 博士, 副教授, 硕士生导师, CCF 会员, 论文通讯作者, 主要研究方向为计算机图形学. 
连续手语识别是一个典型的序列到序列 (sequence to sequence, Seq2Seq)任务, 目标是实现 手语序列到文本序列的转换. 目前, 公开的连续手 语识别数据集都是基于视频的, 如 PHOENIX14 ${ }^{[1]}$, PHOENIX14T ${ }^{[2]}$ 和 $\mathrm{CSL}^{[3]}$. 虽然视频易于获取且包 含丰富的信息，但是对背景、光照和肤色等干扰因 素的鲁棒性较差, 并且处理视频的计算成本较大. 与之相比, 骨架数据能够更加精炼地表达手部信 息, 排除外界因素的干扰, 将模型关注点从传感器 全局视野转移到人体. 手部骨架数据既可以使用 Leap Motion 等三维传感器采集, 也可以通过手势 估计算法从图像或深度图中抽取.

鉴于骨架数据的优点, 本文寻求基于手部骨 架数据的连续手语识别算法的可能性. 针对相关 数据集贵乏的问题, 通过 Leap Motion 收集了一个 基于三维手部骨架的连续手语数据集 LMSLR, 拓 宽了连续手语识别任务的输人数据类型. 受双路 卷积网络 ${ }^{[4]}$ 的启发，从原始数据的帧内和帧间分别 提取人工设计的形状和轨迹, 并通过一系列并行 且共享参数的双路残差网络对形状和轨迹进行优 化和融合, 以得到最终的时空特征序列. 然后, 将 机器翻译中常用的基于注意力机制的编码-解码网 络 ${ }^{[5]}$ 应用到本文任务中, 实现时空特征序列到文本 的映射. 在 LMSLR 数据集上评估本文模型, 并与 基于视频的连续手语识别方法在公共的手语数据 集 $\mathrm{CSL}^{[3]}$ 上进行比较. 实验结果表明, 本文模型具 有良好的性能.

\section{1 相关工作}

随着 Shotton 等 ${ }^{[6]}$ 证明骨架关节的位置、运动 和方向是人类行为的最佳描述符，基于人体骨架 模型的动作识别成为热门研究方向, 并取得一系 列鼓舞人心的结果. 同理, 在手势识别中, 使用骨 架模型表示手部比图像或深度图更加简洁有效. 事实上, de Smedt 等 ${ }^{[7]}$ 论证了这个观点, 同时从 Intel RealSense 获取手部的深度图和 22 关节点骨架 数据, 通过一系列的对比实验证明使用骨架模型表 达手势的优越性. Intel RealSense 和 Leap Motion 等 传感器可以直接获取高精度的三维手部骨架节点信 息. 此外, 手势估计算法可以通过深度图甚至图像 将手部骨架模型的关键点映射到三维或二维坐标系 统 $^{[8-9]}$, 扩展手部骨架数据的来源. 手部骨架数据已 经广泛应用于手势识别和孤立词手语识别. 这是一 项传统的分类任务, 关键是提取可区分的特征描述
符如单指特征 ${ }^{[10]}$ 、双指特征 ${ }^{[11]}$ 和角度特征 ${ }^{[12]}$, 输人 隐条件神经场 ${ }^{[11]}$ 或循环神经网络(recurrent neural

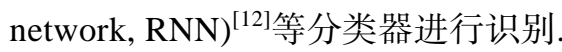

由于缺乏基于手部骨架数据的连续手语数据 集, 已有的连续手语识别工作几乎都是基于视频 的. 目前有 3 种主流的解决方案: 手语序列分割、 联结主义时间分类(connectionist temporal classification, CTC)算法 ${ }^{[13]}$ 和编码-解码网络 ${ }^{[5]}$. 第 1 种方 法是基于孤立识别, 将手语序列分割后逐一识别每 个分割片段对应的孤立词 ${ }^{[1,14]}$. 由于相邻的孤立词 手势之间的过渡动作是模糊的，因此不能保证分割 的准确性. 该解决方案还需要为手语序列的每帧打 上标签, 消耗极大的人力资源. 相比之下, 后 2 种 方法都可以实现输人序列到输出序列的端到端训 练. CTC 算法最早应用于语音识别领域, 使用该算 法的前提条件是输人序列远长于输出序列, 并且输 人-输出序列单调对齐. 连续手语识别同样符合这 个条件. 在一个连续的、未分割的动态手势识别任 务中, Molchanov 等 ${ }^{[15]}$ 将手势序列粗略地划分为若 干片段，每个片段通过卷积神经网络(convolutional neural networks, CNN)提取特征, 进人 RNN 单元, 最后通过 CTC 算法依次输出每个动态手势类别. Camgoz 等 ${ }^{[16]}$ 通过类似的方法翻译未分割的手语 序列, 并在中间表示阶段适当注人专家知识, 实现 良好的翻译效果. 编码-解码网络 ${ }^{[5]}$ 来源于机器翻 译, 通常由 2 组 RNN 充当编码器和解码器. 其中, 编码器将输人序列压缩为一个全局语义向量; 解 码器作为生成模型, 通过语义向量和前一步的输 出逐渐生成目标序列. Bahdanau 等 ${ }^{[17]}$ 首先为编码解码网络引人注意力机制, 通过学习一组权重篮 选出与当前输出单元最相关的输人片段. Camgoz 等 ${ }^{[2]}$ 参考这种结构实现连续手语识别, 其基本思 想是通过 CNN 得到手语视频的特征向量序列, 并 使用基于注意力机制的编码 - 解码网络实现 Seq2Seq 训练与识别. 比较这 3 种解决方案发现, 编码-解码网络既不用进行输人序列的分割, 也没 有 CTC 算法的条件限制, 灵活性和扩展性更好.

\section{2 本文模型}

连续手语识别的目标是学习通过输人的手语 骨架序列 $\boldsymbol{x}=\left\{\boldsymbol{x}_{t} \mid t=1,2, \cdots, T\right\}$ 生成对应的单词序列 $\boldsymbol{y}=\left\{\boldsymbol{y}_{u} \mid u=1,2, \cdots, U\right\}$ 的条件概率 $p(\boldsymbol{y} \mid \boldsymbol{x})$. 其中, $T$ 为手语序列的帧数, $U$ 为输出句子中单词个数. 
在该任务中, $T \gg U$ 且输人序列长达数百帧, 这 使 RNN 等传统序列建模架构的使用变得困难, 如 产生长距离依赖问题. 本文结合人的知识和神经
网络提取原始序列的时空特征, 并将这些特征输 人基于注意力机制的编码-解码网络进行建模和识 别. 其模型框架如图 1 所示.

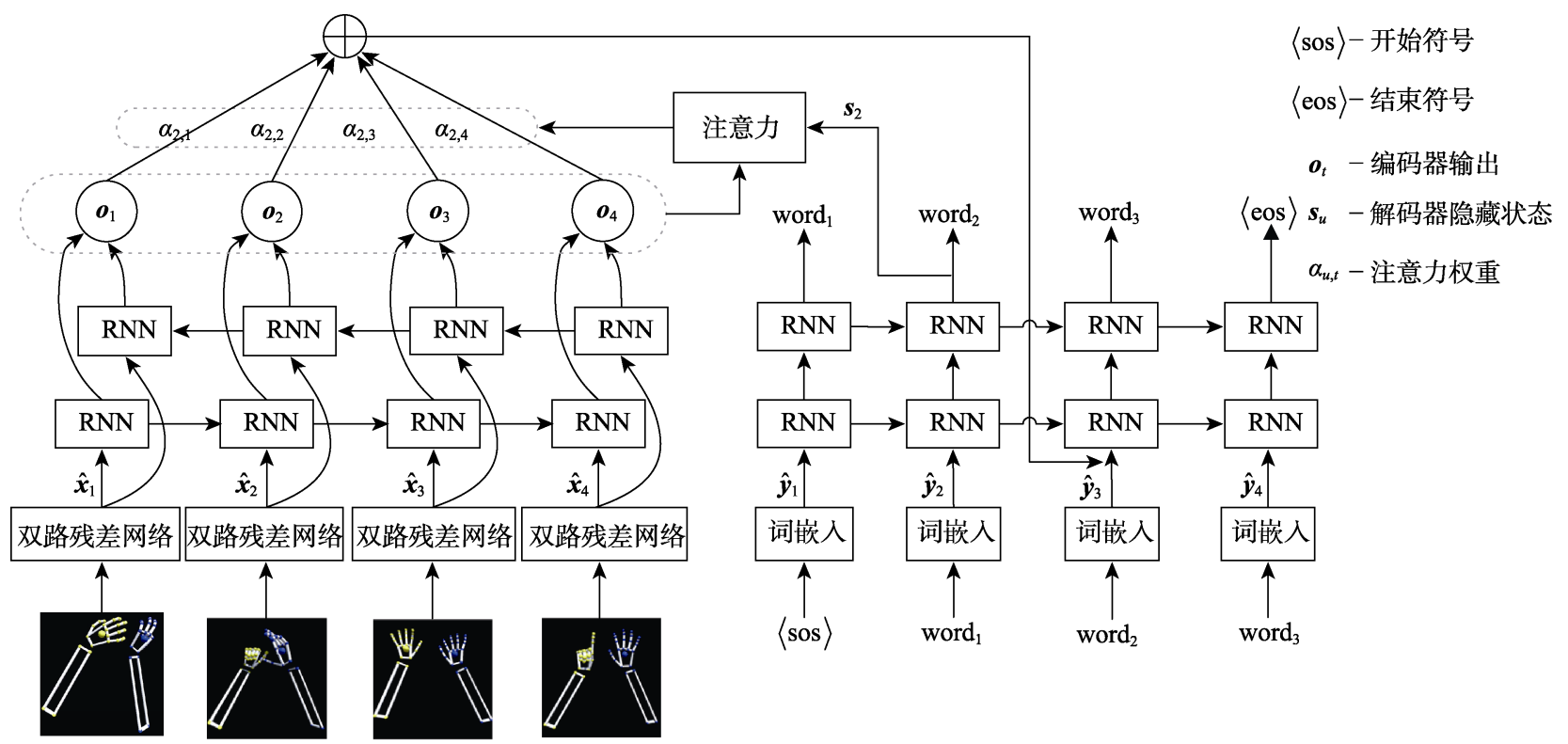

图 1 本文模型框架

\section{1 手部骨架数据描述}

手语使用者在 Leap Motion 上方做手势, Leap Motion 以实测 50 60 帧/s 的速率返回双手的骨架 信息. Leap Motion 拥有区别左右手的能力, 若某 帧只检测到单手，则另一只手的数据用 0 填充，以 确保每帧的数据是完整的. 如图 2 所示, 每只手拥 有 22 个骨架关节点, 包括 20 个手指关节点、 1 个 手腕关节点和一个肘部关节点. 因此, 单帧的初始 骨架数据可以进一步表示为

$$
\boldsymbol{x}_{t}=\left\{\boldsymbol{x}_{t}^{\text {left }}, \boldsymbol{x}_{t}^{\text {right }}\right\}
$$

以左手为例, 进一步划分为

$$
\boldsymbol{x}_{t}^{\text {left }}=\left\{\boldsymbol{x}_{t, m}^{\text {left }} \mid m=1,2, \cdots, 22\right\}
$$

其中, $t$ 和 $m$ 分别表示帧编号和关节点编号; $\boldsymbol{x}_{t, m}^{\text {left }}$ 是一个三元组, 代表第 $t$ 帧左手骨架的第 $m$ 个关节 点在 Leap Motion 坐标系中的三维坐标. 由于左手 和右手的表示和计算是完全相同的，因此下文所 有关于单手数据的描述和计算均以左手为例.

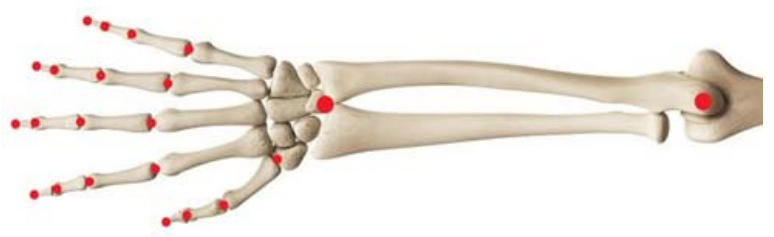

图 2 手部骨架关节点图示

\section{2 人工特征提取}

Leap Motion 对物体的位置非常敏感. 对于计 算机, 手和传感器相对位置的不同会导致同一手 语的不同样本之间存在巨大差异, 即样本离散程 度过高. 这可能会干扰模型的训练, 使其难以收玫 或过拟合. 故需要通过人的经验和知识预先提取 鲁棒的特征, 这有利于聚拢同一个手语的不同样 本, 使模型训练稳定. 手语主要通过双手的手型和 运动轨迹表达, 在此先验知识的基础上, 分别从帧 内和帧间提取手型特征和轨迹特征.

手型特征是为每帧中的每只手建立以腕部为 原点的局部坐标系, 并用局部坐标取代原坐标. 这 种做法可以有效地降低初始样本的离散程度，同 时保留手部形状特征. 手型是在帧内即空间维度 计算的，故可充当空间特征，其计算式为

$$
\boldsymbol{s}_{t, m}^{\text {left }}=\boldsymbol{x}_{t, m}^{\text {left }}-\boldsymbol{x}_{t, 1}^{\text {left }}
$$

其中, $\boldsymbol{x}_{t, 1}^{\mathrm{left}}$ 为手腕坐标. 使用相同的方式处理右手 可得到第 $t$ 帧双手骨架数据的手型特征向量 $\boldsymbol{s}_{t}$.

双手从全局坐标系转移到局部坐标系的变换 使手型对手与传感器的相对位置具有鲁棒性, 但 会丢失手在时间维度的运动信息, 如手的抖动和 移动等. 因此，以前后 2 帧对应关节点之间的位移 向量作为轨迹特征, 可以有效地弥补帧间手部相 对位置信息的缺失. 由于该特征是在相邻帧之间 
计算的，也就是跨时间维度的计算，故可以充当时 间特征，其计算式为

$$
\boldsymbol{I}_{t, m}^{\text {left }}=\boldsymbol{x}_{t, m}^{\text {left }}-\boldsymbol{x}_{t-1, m}^{\text {left }}
$$

最终得到第 $t$ 帧双手骨架数据的轨迹特征向量 $\boldsymbol{I}_{t}$.

\section{3 双路残差网络}

神经网络通过堆叠隐藏层, 每层都是对上一 层的输出实施进一步计算, 逐步得到对模型而言 更高级的抽象特征. 第 2.2 节中, 通过人的知识提 取的手型和轨迹特征对于模型是低层次的, 因此 利用多层前馈全连接网络可使人工特征转化为更 抽象的特征, 并促进手型和轨迹特征的融合. 为了 解决网络退化问题, 构造如图 3 所示双路残差网络. 其中, 每路分别对手型特征和轨迹特征进行优化, 残差块使用跳跃连接, 使网络训练更稳定 ${ }^{[18]}$. 令该 网络输人为 $\boldsymbol{x}_{\mathrm{res}}$, 输出为 $\boldsymbol{y}_{\mathrm{res}}$, 其数学表达式为

$$
\boldsymbol{y}_{\text {res }}=\mathcal{F}\left(\boldsymbol{x}_{\text {res }}\right)+\boldsymbol{x}_{\text {res }}
$$

其中, $\mathcal{F}$ 为隐藏层和激活函数的组合. 本文利用 双路残差网络计算第 $t$ 帧的最终特征向量, 即

$$
\hat{\boldsymbol{x}}_{t}=\mathcal{C}\left(\mathcal{F}_{1}\left(\boldsymbol{s}_{t}\right)+\boldsymbol{s}_{t}, \mathcal{F}_{2}\left(\boldsymbol{I}_{t}\right)+\boldsymbol{I}_{t}\right)
$$

其中, $\mathcal{F}_{1}$ 和 $\mathcal{F}_{2}$ 包含 2 层前馈全连接网络和 $\operatorname{ReLU}$ 非 线性激活函数 ${ }^{[19]} ; \mathcal{C}$ 表示 2 个向量的拼接. 这种残 差块的跳跃连接可以有效地解决网络退化问题 ${ }^{[18]}$. 此外，分路处理不同特征的思想 ${ }^{[4]}$ 允许模型学习更 多细节，可提高特征的细淢度，使不同的特征达到 更好的互补效果。

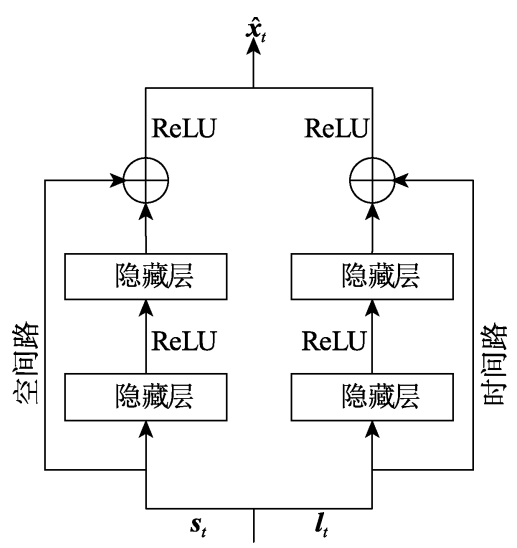

图 3 双路残差网络结构

\section{4 词嵌入表示}

神经机器翻译方法从源序列和目标序列的标 记化人手，通过词嵌人 ${ }^{[20]}$ 将它们投影到连续的空 间中. 本文任务只需要将目标文本序列转换为模 型中使用的向量. 对于词嵌人，首先给每个孤立词 设置一个编号, 并将其转换成独热向量; 接着使用
全连接层将孤立词的独热向量向更密集的空间进 行线性投影. 这是一个稠密矩阵 $\boldsymbol{W}_{\mathrm{emb}}$ (权重矩阵) 乘以一个稀疏向量 $\boldsymbol{y}_{u}$ (独热向量)得到词嵌人向量 $\hat{\boldsymbol{y}}_{u}$ (密集向量)的过程, 表示为

$$
\hat{\boldsymbol{y}}_{u}=\boldsymbol{W}_{\mathrm{emb}} \cdot \boldsymbol{y}_{u}
$$

由于独热向量中只有单个元素为 1 , 其余元素 均为 0 , 因此返回的结果为权重矩阵的某行. 使用 词嵌人的主要思想是将稀疏的向量表示转换为某 种更密集的形式，其中意思相近的词语对应的词 向量之间的几何距离也更近.

\section{5 基于注意力机制的编码-解码网络}

\subsection{1 编码-解码网络}

基于 RNN 结构的编码-解码网络 ${ }^{[5]}$ 已经成功地 取代了传统的基于语料库的机器翻译方法, 成为 一种流行的神经机器翻译方法. 这种网络结构也 适用于本文任务, 它等价于一个映射函数, 通过并 行双路残差网络输出的手语特征序列 $\hat{x}=$ $\left\{\hat{\boldsymbol{x}}_{t} \mid t=1,2, \cdots, T\right\}$, 生成目标句子的词嵌人向量序 列 $\hat{\boldsymbol{y}}=\left\{\hat{\boldsymbol{y}}_{u} \mid u=1,2, \cdots, U\right\}$, 最终实现连续手语识别 的目的.

编码-解码网络由一个编码器和一个解码器组 成, 通常使用 2 组 RNN 分别充当编码器和解码器. 编码器依次读取手语特征序列, 将其编码为全局 语义向量 $\boldsymbol{c}$. 整个过程表示为

$$
\begin{gathered}
\boldsymbol{h}_{t}=f\left(\hat{\boldsymbol{x}}_{t}, \boldsymbol{h}_{\mathrm{t}-1}\right) \\
\boldsymbol{c}=q\left(\boldsymbol{h}_{\mathrm{l}: T}\right)
\end{gathered}
$$

其中, $\boldsymbol{h}_{t}$ 为 $t$ 时刻的编码器 RNN 单元隐藏状态; $f$ 和 $q$ 代表某些非线性函数. 例如, $f$ 是一个长 短期记忆网络(long short term memory, LSTM)或门 控循环单元(gated recurrent unit, GRU), $q$ 可以简 单地定义为取最后一个 RNN 单元的隐藏状态, 即 $q\left(\boldsymbol{h}_{1: T}\right)=\boldsymbol{h}_{T}$.

整个解码过程从输人句子的开始符号 $\langle\mathrm{sos}\rangle$ 持 续到输出句子的结束符号 $\langle\mathrm{eos}\rangle$. 解码器一般通过 所有先前预测的词 $\hat{\boldsymbol{y}}_{1: u-1}$ 和语义向量 $\boldsymbol{c}$ 预测当前词 $\hat{\boldsymbol{y}}_{u}$. 换言之, 解码器通过逐词生成句子, 将联合 概率分解为有序条件概率, 以此计算出翻译 $\hat{\boldsymbol{y}}$ 的 概率，即

$$
p(\hat{\boldsymbol{y}})=\prod_{u=1}^{U} p\left(\hat{\boldsymbol{y}}_{u} \mid \hat{\boldsymbol{y}}_{1: u-1}, \boldsymbol{c}\right)
$$

更详细地，可以将每个条件概率建模为

$$
p\left(\hat{\boldsymbol{y}}_{u} \mid \hat{\boldsymbol{y}}_{1: u-1}, \boldsymbol{c}\right)=g\left(\hat{\boldsymbol{y}}_{u-1}, \boldsymbol{s}_{u}, \boldsymbol{c}\right)
$$


其中, $s_{u}$ 为解码器在 $u$ 时刻的隐藏状态; $g$ 为输出 $\hat{y}_{u}$ 概率的解码器隐藏层和非线性激活函数的组合.

\section{5 .2 注意力机制}

编码-解码网络将整个输人序列压缩成一个固 定维数的语义向量 $\boldsymbol{c}$. 当输人序列较长时, $\boldsymbol{c}$ 无法 存储足够的信息. 注意力机制允许解码器的每步 对输人序列中与当前预测最相关的一部分进行篮 选, 而不再需要将所有信息存储在语义向量 $\boldsymbol{c}$. 以 翻译第 $u$ 个词为例, 解码器将对应时间步的隐藏 状态 $s_{u}$ 作为查询输人对编码器每步的输出 $\boldsymbol{o}_{1} \sim \boldsymbol{o}_{T}$ 进行轮询, 并计算出一组反映与查询输人相关程 度的权重 $\alpha_{u, 1} \sim \alpha_{u, T}$; 再根据这组权重对编码器的 输出加权求和得到语义向量 $\boldsymbol{c}_{u}$, 表示与翻译当前 词最相关的输人序列信息. 整个计算过程表示为

$$
\begin{gathered}
\boldsymbol{c}_{u}=\sum_{t=1}^{T} \alpha_{u, t} \boldsymbol{o}_{t} \\
\alpha_{u, t}=\frac{\exp \left(e\left(\boldsymbol{o}_{t}, \boldsymbol{s}_{u}\right)\right)}{\sum_{i=1}^{T} \exp \left(e\left(\boldsymbol{o}_{i}, \boldsymbol{s}_{u}\right)\right)}
\end{gathered}
$$

其中, $\boldsymbol{o}_{t}$ 为编码器第 $t$ 步的输出; $s_{u}$ 为解码器预测 第 $u$ 个单词相关的解码器隐藏状态; $e(\boldsymbol{o}, \boldsymbol{s})$ 为计算 输人序列的每个片段与当前解码器隐藏状态相关 性的函数, 最常见的是用于前馈神经网络 ${ }^{[17]}$ 或基 于矩阵乘法的方法 ${ }^{[21]}$, 即

$$
e(\boldsymbol{o}, \boldsymbol{s})= \begin{cases}\boldsymbol{V}^{\mathrm{T}} \tanh (\boldsymbol{W}[\boldsymbol{o}, \boldsymbol{s}]), & \text { 文献[17] } \\ \boldsymbol{o}^{\mathrm{T}} \boldsymbol{W}, & \text { 文献[21] }\end{cases}
$$

其中, $\boldsymbol{V}$ 和 $\boldsymbol{W}$ 为权重矩阵.

值得注意的是, 与传统的编码-解码网络相比, 除了增加注意力机制外, 本文编码-解码网络还有 2 个不同之处: (1) 编码器使用双向 RNN, 这是因 为解码器在通过注意力机制查询原始输人序列的 某个帧时，通常还需要知道该帧的上下文信息; (2) 注意力机制将编码器与解码器断开, 解码器仅依 靠注意力机制获得输人序列的信息, 断开编码器 和解码器的连接允许它们自由选择各自的实现模 型，如具有不同层次、维度或结构的 RNN.

\section{3 实验结果与分析}

使用 TensorFlow 框架构建模型, 硬件上使用 GeForce GTX 1070Ti 显卡加速训练. 双路残差网 络每路的隐藏层和编码-解码网络双层结构中每个 RNN 单元的大小均设置为 256. 训练目标是通过
迭代训练使对数困惑度损失函数最小化, 即

$$
\mathcal{F}_{\text {loss }}=-\frac{1}{U} \sum_{u=1}^{U} \ln \left(p\left(\hat{\boldsymbol{y}}_{u} \mid \hat{\boldsymbol{y}}_{1: u-1}, \boldsymbol{c}_{u}\right)\right)
$$

该损失函数相当于预测分布和参考分布之间的交 叉熵. 将学习率设为 0.0001 , 采用小批量算法训练 模型, 每个小批量包含 100 个实例, 迭代轮次为 10 , 并使用 Adam 优化器对梯度下降算法进行优 化, 使模型参数稳定. 准确率 $a$ 用于度量预测句子 与参考句子之间的相似度，其作为连续手语识别 中常用的性能评估指标，计算式为

$$
a=1-\frac{S+D+I}{N} \times 100 \%
$$

其中, $S, D$ 和 $I$ 分别为将预测句子转换为参考句 子所需的替换、删除和添加操作的最少次数; $N$ 为 参考句子中的单词个数.

\section{1 数据集}

本文实验使用 2 个连续手语数据集：(1) 基于 三维手部骨架的 LMSLR 数据集; (2) 公开的基于 视频的 CSL 数据集 ${ }^{[3]}$. 由于手语识别中还没有公 开的基于手部骨架的连续手语数据集，因此本文 利用 Leap Motion 传感器收集数据构建 LMSLR 数 据集, 其详细的信息统计如表 1 所示. 该数据集包 括训练本文模型所需的骨架关节坐标, 以及手掌 中心坐标和手掌平面法向量等. 掌心的数据也是 常用的手部信息, 用于计算部分人工特征. 10 位手 语使用者参与了这个数据集的收集工作, 每人贡 献 1000 个手语样本. 其中 8 人的样本充当训练集, 1 人的样本用于验证集, 1 人的样本用于测试集.

\section{表 1 LMSLR 数据集统计}

\begin{tabular}{lc}
\hline \multicolumn{1}{c}{ 项目 } & 参数 \\
\hline 样本量 & 10000 \\
词汇量 & 298 \\
句子量 & 100 \\
最大/最小/平均手语序列长度 & $443 / 73 / 215.3$ \\
最大/最小/平均句子长度 & $12 / 4 / 7.8$ \\
\hline
\end{tabular}

CSL 数据集 ${ }^{[3]}$ 由微软 Kinect 摄像头记录，提供 RGB 信息、深度信息和肢体骨架信息. 本文实验取 RGB 信息. CSL 数据集拥有 25000 个带标签的视频 样本, 包含 50 位手语使用者总计超过 $100 \mathrm{~h}$ 的视频 片段, 其中词汇量和句子量分别为 178 和 100 . 每 个视频样本都由专业的手语教师用完整的句子进 行注释, 其中选择 17000 个样本进行训练, 2000 个 样本进行验证, 剩下的 6000 个样本进行测试. 同 一位手语使用者提供的样本不会同时出现在训练 
集和测试集中.

\section{2 单因素实验}

在 LMSLR 数据集上通过单因素实验研究 3 种 因素的不同选择对准确率的影响, 以达到最佳的 识别效果. 3 种因素分别为帧采样方式的选择、 RNN 单元的选择和注意力机制的选择. 假设这些 因素是相互独立的，对某个因素进行实验时，将其 他因素固定. 通过验证集上的识别准确率确定每 个因素的具体设定，并在其他单因素实验以及下 文实验中沿用最佳的设定.

\subsection{1 帧采样方式的选择}

在一个手语识别系统中，知道最佳的帧采样 方式有助于通过跳过不必要的帧降低推理引擎的 计算成本，同时避免以牺牲准确率为代价。在初始 序列上采用均匀采样的方法提取帧，具体分为固 定帧数的均匀采样和固定间隔的均匀采样. 前者 为均匀地提取固定数量的帧数, 如 10 帧、 20 帧; 而 后者每间隔固定的帧数提取一帧，如每 2 帧取一 帧、每 3 帧取一帧. 表 2 展示不同的帧采样方式在 验证集和测试集上的准确率比较.

表 2 帧采样方式的准确率评估 $\%$

\begin{tabular}{cccc}
\hline 帧采样方式 & 帧数 & 验证集 & 测试集 \\
\hline & 10 & 87.69 & 87.83 \\
固定帧数 & 20 & 89.21 & 88.89 \\
& 30 & 90.15 & 89.86 \\
& 40 & 91.56 & 91.14 \\
& 50 & 91.51 & 91.74 \\
\hline \multirow{6}{*}{ 固定间隔 } & 2 & 93.62 & 93.09 \\
& 3 & 93.70 & 93.75 \\
& 4 & 92.84 & 92.93 \\
& 6 & 94.70 & 94.04 \\
& 7 & 91.47 & 90.98 \\
& 8 & 90.81 & 90.60 \\
\hline
\end{tabular}

由于固定帧数的采样方式难以区分长短句, 因此一般情况下固定间隔的采样方式能得到更高 的准确率. 在固定间隔的采样方式中，每 5 帧取一 帧的方式在验证集和测试集上都达到最高的准确 率, 此时样本的平均长度为 43 帧, 在计算成本和 识别精度之间得到较好的平衡.

\subsubsection{RNN 单元的选择}

RNN 能有效地对序列进行建模，但随着序列 长度的增加, 它在处理长期记忆方面存在缺陷. LSTM 和 GRU 优化传统 RNN 单元，都能对有价值 的信息进行长期记忆. 使用 LSTM 和 GRU 训练了
2 个带注意力机制的编码-解码网络, 以确定选择 哪种递归单元，它们的其他参数设定是完全相同 的. 从表 3 可以看出, GRU 训练的网络在准确率上 优于 LSTM. 这是由于 LSTM 单元中附加的参数和 训练数据有限造成过拟合. 与 LSTM 相比, GRU 参 数更少，收敛速度更快，且不容易发生过拟合.

表 3 循环单元的准确率评估

\begin{tabular}{ccc}
\hline 循环单元 & 验证集 & 测试集 \\
\hline LSTM & 89.23 & 88.39 \\
GRU & 94.70 & 94.04 \\
\hline
\end{tabular}

\subsection{3 注意力机制的选择}

本节评估不同注意力机制对连续手语识别任 务的影响. 选择第 2.5.2 节中描述的基于前馈神经 网络的注意力 ${ }^{[17]}$ 和基于矩阵乘法的注意力 ${ }^{[21]}$, 这 2 种经典注意力机制的思想大致相同，仅在计算方 式和输人输出上略有不同. 为了显示注意力机制 的优势, 本文额外训练一个不带任何注意力机制 的普通编码-解码网络. 实验结果如表 4 所示, 首 先观察到, 加人注意力机制后, 准确率有很大的提 高, 这是因为普通的编码-解码网络在输人序列很 长的情况下严重丢失信息. 比较注意力机制时, 在 本文实验中, 基于前馈神经网络的注意力 ${ }^{[17]}$ 无论 在验证集还是在测试集上都取得更优的结果.

表 4 注意力机制的准确率评估 $\%$

\begin{tabular}{lcc}
\hline \multicolumn{1}{c}{ 注意力机制 } & 验证集 & 测试集 \\
\hline 无 & 82.35 & 82.23 \\
基于前馈神经网络 $^{[17]}$ & 94.70 & 94.04 \\
基于矩阵乘法 $^{[21]}$ & 89.72 & 89.36 \\
\hline
\end{tabular}

\section{3 人工特征评估}

本节实验在 LMSLR 数据集上评估不同人工设 计的特征表现. 从原始数据中计算出相应的人工 设计的特征向量序列, 并将其直接输人基于注意 力机制的编码-解码网络模型中, 免除双路残差网 络对人工特征进行优化和融合. 将本文提出的手 型特征、轨迹特征以及二者的融合与单指特征 ${ }^{[10]}$ 、 单指 + 双指特征 ${ }^{[11]}$ 、角度特征 ${ }^{[12]}$ 以及原始数据进行 比较, 实验结果如表 5 所示. 虽然手指或角度特征 适用于描述手指间相对运动较多的动态手势, 但 它们的计算仅使用非常有限的指尖和掌心信息, 不足以描述更复杂的连续手语, 所以准确率甚至 低于原始数据. 原始数据包含完整的手部关节点 在 Leap Motion 坐标系中的三维坐标信息. 然而手 
在 Leap Motion 坐标系中的高度和位置的不同会对 模型判断带来干扰，所以准确率并不高.

表 5 人工特征的准确率比较

$\%$

\begin{tabular}{lc}
\hline \multicolumn{1}{c}{ 人工特征 } & 测试集 \\
\hline 单指特征 $^{[10]}$ & 56.99 \\
单指+双指特征 $^{[11]}$ & 65.20 \\
角度特征 $^{[12]}$ & 75.08 \\
原始数据 $^{\text {手型特征 }}$ & 78.76 \\
轨迹特征 & 92.47 \\
手型+轨迹特征 & 84.75 \\
\hline
\end{tabular}

与其他文献设计的特征和原始骨架数据相比, 本文提出的手型特征包含整只手的主要关节信息, 避免局限于指尖或个别关节点，对手在全局的位 置也不敏感. 轨迹特征描述各关节点在时间维度 的变化, 以弥补运动信息的丢失. 这 2 个特征很好 地涵盖整个手语序列的时空特征, 实验结果也进 一步表明, 它们在连续手语识别任务中都优于其 他常用特征, 手型特征达到最佳的准确率. 此外, 这 2 个特征起到互补的作用, 将它们融合后, 准确 率得到进一步提高.

\section{4 双路残差网络评估}

本节评估双路残差网络对特征优化和融合的 意义. 设计 3 种基准方法进行比较：双路前馈全连 接网络、单路前馈全连接网络和单路残差网络. 具 体来说, 单路的方式是将手型和轨迹特征融合后 一起输人单路的网络进行特征优化; 前馈全连接 网络取消残差网络中的跳跃连接. 为了使单路和 双路 2 种模式的网络参数量相同以保证公平性，单 路的网络中各隐藏层节点数调整为 512 . 特征的融 合采用的是向量拼接的方式. 测试集上的准确率 如表 6 所示.

\section{表 6 单路与双路不同残差网络的准确率评估}

$\%$

\begin{tabular}{lc}
\hline \multicolumn{1}{c}{ 网络 } & 测试集 \\
\hline 单路前馈全连接 & 92.46 \\
双路前馈全连接 & 92.95 \\
单路残差 & 93.47 \\
双路残差 & 94.04 \\
\hline
\end{tabular}

从特征优化的角度,一个有趣的现象是, 使用 普通前馈全连接网络之后, 无论是单路还是双路 的处理都反而使准确率下降. 这是由网络退化引 起的问题，幸运的是残差网络可以确保模型的性 能不下降且有助于模型优化 ${ }^{[18]}$. 实验结果表明,
利用残差网络优化特征后, 准确率有所提高. 对特 征尝试前融合和后融合 2 种模式, 前者采用单路 网络同时优化融合后的特征，后者采用双路网络 分别优化 2 种特征后再将其融合. 相比之下, 后 融合的方式表现更好，因为它可以分别处理空间 和时间维度，提高特征细淢度. 综上，双路残差 网络无论是对特征的优化还是融合，都发挥了积 极的作用.

\subsection{CSL 数据集上的比较}

为了验证本文模型对不同来源的骨架数据的 适用性, 将模型迁移到基于视频的连续手语数据 集 $\mathrm{CSL}^{[3]}$. 使用 OpenPose 库从 CSL 抽取人体关键 点的坐标数据, 其中包含手部、肢体和脸部的关键 点. 本文只使用手部和肘部的 22 个骨架关节点数 据训练模型. 表 7 给出本文模型和其他文献模型的 比较, 结果显示, 本文模型的准确率达到 $97.2 \%$, 逼近 Zhou 等 ${ }^{[22]}$ 提出的基于多线索的模型，并大幅 领先于其他模型. 这表明, 虽然通过手势估计从视 频得到的骨架数据不如从传感器直接得到的那么 精确, 但是使模型的关注点从整个图像聚焦到关 键的手部，对于手语识别是有效的.

表 7 不同模型在 CSL 数据集上的准确率比较 \%

\begin{tabular}{lc}
\hline \multicolumn{1}{c}{ 模型 } & 准确率 \\
\hline LS-HAN $^{[4]}$ & 82.7 \\
HLSTM $^{[23]}$ & 89.8 \\
CTF $^{[24]}$ & 88.8 \\
DenseTCN $^{[25]}$ & 85.7 \\
STCM $^{[22]}$ & 97.9 \\
本文 & 97.2 \\
\hline
\end{tabular}

值得注意的是, 本文模型没有借助表情和肢 体对手语表达的辅助作用, 而是只利用在手语表 达中起决定性作用的双手信息达到较高的准确率, 充分利用辅助信息也许可以进一步提高识别性能. 另外, 本文通过模型浮点运算次数(floating point of operations, FLOPs) 衡量时间复杂度, 通常 FLOPs 越小, 实时性更好. 得益于低圥余的骨架数 据, 本文模型所需要的硬件算力仅为约 $6.3 \mathrm{G}$ FLOPs，低于文献[22]中的 10.3G FLOPs.

\section{6 翻译示例分析}

除了采用准确率指标对连续手语识别性能进 行评估以外, 更加直观的判定方式是检查翻译结 果. 为了便于理解和分析, 表 8 展示了本文模型在 LMSLR 数据集上进行测试时挑选的 5 条具有代表 性的翻译示例. 
表 8 本文模型在 LMSLR 数据集上翻译示例

\begin{tabular}{cll}
\hline 示例 & \multicolumn{1}{c}{ 预测句子 } & \multicolumn{1}{c}{ 参考句子 } \\
\hline 1 & 你几岁?? & 你几岁? \\
2 & 有去北京的飞机票? & 有去北京的飞机票吗? \\
3 & 明天见, 等等会见. & 明天见, 等会儿见. \\
4 & 试衣间在那里. & 试衣间在那里. \\
5 & 洗手间在哪里? & 洗手间在哪里? \\
\hline
\end{tabular}

示例 1 示例 3 是没有实现完美识别的例子. 不难发现，即使没有达到 100.0\%的准确率，大多 数情况下只是少量的多词(示例 1)、缺词(示例 2) 或错词 (示例 3), 并不会影响人们对句子的理解. 另外, 在示例 4 和示例 5 中, “那里”和“哪里”的手语 表达是完全相同的, 本文模型可以根据语境准确 地输出合适的词.

\section{4 结 语}

本文提出一种基于三维手部骨架数据的连续 手语识别模型, 可用于识别多种途径获得的手语 骨架序列并将其翻译为文本, 具有较高的准确率 以及较广的适用性. 通过 Leap Motion 收集了一个 用三维手部骨架数据表示的连续手语数据集, 并 准备在全球范围内共享, 惠及手语研究者.

未来的工作可以从 3 个方面人手: (1) 充分发 挥表情和肢体对手语表达的辅助作用, 并研究非 手部的信息对手语识别的影响；(2) 骨架模型是一 个稳定的拓扑结构，关节点之间具有固定的连接 关系，在提取时空特征时可以将感受野从局部慢 慢扩大到全局，这样的做法非常有助于学习层次 化特征; (3) 建立更加详尽和完备的连续手语识别 语料库和数据集, 推动手语识别的研究.

\section{参考文献(References):}

[1] Koller O, Forster J, Ney H. Continuous sign language recognition: towards large vocabulary statistical recognition systems handling multiple signers[J]. Computer Vision and Image Understanding, 2015, 141(11): 108-125

[2] Camgoz N C, Hadfield S, Koller O, et al. Neural sign language translation[C] //Proceedings of the IEEE Computer Society Conference on Computer Vision and Pattern Recognition. Los Alamitos: IEEE Computer Society Press, 2018: 7784-7793

[3] Huang J, Zhou W G, Zhang Q L, et al. Video-based sign language recognition without temporal segmentation[C] //Proceedings of the AAAI Conference on Artificial Intelligence. Palo Alto: AAAI Press, 2018: 2257-2264

[4] Simonyan K, Zisserman A. Two-stream convolutional networks for action recognition in videos[OL]. [2020-11-30]. https://arxiv.org/abs/1406.2199

[5] Cho K, Merrienboer B V, Gulcehre C, et al. Learning phrase representations using RNN encoder-decoder for statistical machine translation[OL]. [2020-11-30]. https://arxiv.org/abs/1406. 1078

[6] Shotton J, Fitzgibbon A, Cook M, et al. Real-time human pose recognition in parts from single depth images[C] //Proceedings of the IEEE Computer Society Conference on Computer Vision and Pattern Recognition. Los Alamitos: IEEE Computer Society Press, 2011: 1297-1304

[7] de Smedt Q, Wannous H, Vandeborre J P. Skeleton-based dynamic hand gesture recognition[C] //Proceedings of the IEEE Computer Society Conference on Computer Vision and Pattern Recognition Workshops. Los Alamitos: IEEE Computer Society Press, 2016: 1206-1214

[8] Wan C D, Probst T, van Gool L, et al. Dense 3D regression for hand pose estimation[C] //Proceedings of the IEEE Computer Society Conference on Computer Vision and Pattern Recognition. Los Alamitos: IEEE Computer Society Press, 2018: 5147-5156

[9] Zimmermann C, Brox T. Learning to estimate 3D hand pose from single RGB images[C] //Proceedings of the IEEE International Conference on Computer Vision. Los Alamitos: IEEE Computer Society Press, 2017: 4913-4921

[10] Marin G, Dominio F, Zanuttigh P. Hand gesture recognition with jointly calibrated leap motion and depth sensor[J]. Multimedia Tools and Applications, 2016, 75(22): 14991-15015

[11] Lu W, Tong Z, Chu J H. Dynamic hand gesture recognition with leap motion controller[J]. IEEE Signal Processing Letters, 2016, 23(9): 1188-1192

[12] Avola D, Bernardi M, Cinque L, et al. Exploiting recurrent neural networks and leap motion controller for sign language and semaphoric hand gestures[J]. IEEE Transactions on Multimedia, 2019, 21(1): 234-245

[13] Graves A, Fernández S, Gomez F, et al. Connectionist temporal classification: Labelling unsegmented sequence data with recurrent neural networks[C] //Proceedings of International Conference on Machine Learning. New York: ACM Press, 2006: 369-376

[14] Wu D, Pigou L, Kindermans P J, et al. Deep dynamic neural networks for multimodal gesture segmentation and recognition[J]. IEEE Transactions on Pattern Analysis and Machine Intelligence, 2016, 38(8): 1583-1597

[15] Molchanov P, Yang X D, Gupta S, et al. Online detection and classification of dynamic hand gestures with recurrent 3D convolutional neural networks[C] //Proceedings of the IEEE Computer Society Conference on Computer Vision and Pattern Recognition. Los Alamitos: IEEE Computer Society Press, 2016: 4207-4215

[16] Camgoz N C, Hadfield S, Koller O, et al. SubUNets: end-to-end hand shape and continuous sign language recognition[C] //Proceedings of the IEEE International Conference on Computer Vision. Los Alamitos: IEEE Computer Society Press, 2017: 3075-3084

[17] Bahdanau D, Cho K, Bengio Y. Neural machine translation by jointly learning to align and translate[OL]. [2020-11-30]. 
https://arxiv.org/abs/1409.0473

[18] He K M, Zhang X Y, Ren S Q, et al. Deep residual learning for image recognition[C] //Proceedings of the IEEE Computer Society Conference on Computer Vision and Pattern Recognition. Los Alamitos: IEEE Computer Society Press, 2016: 770-778

[19] Nair V, Hinton G E. Rectified linear units improve restricted Boltzmann machines[C] //Proceedings of the International Conference on Machine Learning. New York: ACM Press, 2010: 807-814

[20] Mikolov T, Chen K, Corrado G, et al. Efficient estimation of word representations in vector space[OL]. [2020-11-30]. https://arxiv.org/abs/1301.3781

[21] Luong M T, Pham H, Manning C D. Effective approaches to attention-based neural machine translation[C] //Proceedings of the Conference on Empirical Methods in Natural Language Processing. Stroudsburg: ACL Press, 2015: 1412-1421
[22] Zhou H, Zhou W G, Zhou Y, et al. Spatial-temporal multi-cue network for continuous sign language recognition[C] //Proceedings of the AAAI Conference on Artificial Intelligence. Palo Alto: AAAI Press, 2020: 13009-13016

[23] Guo D, Zhou W G, Li H Q, et al. Hierarchical LSTM for sign language translation[C] //Proceedings of the AAAI Conference on Artificial Intelligence. Palo Alto: AAAI Press, 2018: 6845-6852

[24] Wang S, Guo D, Zhou W G, et al. Connectionist temporal fusion for sign language translation[C] //Proceedings of the ACM International Conference on Multimedia. New York: ACM Press, 2018: 1483-1491

[25] Guo D, Wang S, Tian Q, et al. Dense temporal convolution network for sign language translation[C] //Proceedings of the International Joint Conference on Artificial Intelligence. Palo Alto: AAAI Press, 2019: 744-750 